\title{
Modelado 3D como herramienta educacional para el desarrollo de competencias de los nuevos grados de Bellas Artes
}

\section{D Modeling as an educational tool for the development of skills of the new degrees in Arts}

\author{
JoRge DE LA TORRe CANTERO \\ Universidad de La Laguna. La Laguna. España. \\ Departamento de Expresión Gráfica en Arquitectura e Ingeniería. \\ jcantero@ull.es \\ Jose LUIS SAORÍN \\ Universidad de La Laguna. La Laguna. España. \\ Departamento de Expresión Gráfica en Arquitectura e Ingeniería. \\ jlsaorin@ull.es \\ Carlos Carbonell \\ Universidad de La Laguna. La Laguna. España. \\ Departamento de Expresión Gráfica en Arquitectura e Ingeniería. \\ ccarbone@ull.es \\ Ma Dolores del Castillo Cossío \\ Universidad de La Laguna. La Laguna. España. Departamento de Dibujo, Diseño y Estética. \\ info@1oladelcastillo.es

\section{Manuel Contero} \\ Universidad Politécnica de Valencia. Valencia. España. \\ Instituto en Bioingeniería y Tecnología orientada al ser humano (I3BH). \\ mcontero@labdesign.i3bh.es
}

Recibido: 27 de junio de 2011

Aprobado: 9 de enero de 2012

\section{Resumen}

En este artículo se describe un estudio piloto consistente en la realización de un taller de modelado 3D como herramienta de innovación educativa para el desarrollo de competencias contempladas en los nuevos grados de Bellas Artes.

Entre ellas, el Espacio Europeo de Educación Superior, propone la competencia de elegir el sistema de representación adecuado, la utilización de herramientas tecnológicas avanzadas y el desarrollo de la capacidad de visión espacial. El estudio ha sido realizado con estudiantes de Grado en Bellas Artes de la Universidad de La Laguna durante el curso académico 2010-2011. El software elegido para el taller ha sido Google Sketchup 8, en su versión gratuita. Para medir la influencia que ha tenido la realización del mismo los alumnos rellenaron una encuesta de satisfacción sobre el taller, y se les midió el efecto sobre la visión espacial mediante el Mental Rotation Test (MRT). Los resultados indican que este tipo de iniciativas permiten mejorar las competencias de los estudiantes.

Palabras Clave: Competencias, Espacio Europeo de Educación Superior, Innovación Educativa, Nuevas Tecnologías, Visión Espacial. 
De la Torre Cantero, J. Saorín, J.L. Carbonell, C. Del Castillo Cossío, M.D. y Contero, M. (2012): Modelado 3d como herramienta educacional para el desarrollo de competencias de los nuevos grados de Bellas Artes. Arte, Individuo y Sociedad, 24 (2) 179-193

\begin{abstract}
This article describes a pilot study involving the realization of a 3D modelling workshop as an innovative educational tool for development skills contemplated by the new degrees in Arts. The new European Higher Education Area includes the development of abilities, such us the capacity to choose the appropriate representation system, using advanced technological tools and developing the capacity for spatial vision. The study was conducted with students in Arts Degree from the University of La Laguna during the academic year 2010-2011. The software chosen for the workshop has been Google Sketchup 8, free version. To measure the results of the pilot study, students completed a survey of effectiveness, efficiency and satisfaction on the Workshop, and it was measured the effect of spatial vision using the Mental Rotation Test (MRT). The results show that this kind of initiatives allow to improve the skills of students.
\end{abstract}

Key Words: Skills, European Higher Education Area, Educational Innovation, New Technologies, Spatial Abilities.

De la Torre Cantero, J. Saorín, J.L. Carbonell, C. Del Castillo Cossío, M.D. Contero, M. (2012): 3D Modeling as an educational tool for the development of skills of the new degrees in Arts. Arte, Individuo y Sociedad, 24 (2) 179-193

Sumario: 1. Introducción, 2. Antecedentes, 3. Las habilidades espaciales y su medición, 4. Prueba piloto: Taller de modelado 3D, 4.1. Participantes, 4.2. Hardware y software, 4.3. Medida, 4.4. Instrucción, 4.5. Hipótesis de trabajo, 5. Análisis de los datos, 5.1. Habilidades espaciales, 5.2. Aprendizaje de Sistemas de Representación, 5.3. Empleo de nuevas herramientas y motivación, 6. Conclusiones y futuros trabajos. Referencias.

\title{
1. Introducción
}

En el marco del Espacio Europeo de Educación Superior, el objetivo principal del proceso de enseñanza-aprendizaje consiste no solo en la adquisición de conocimientos por parte del alumno, sino en el desarrollo de una serie de competencias (capacidades y destrezas) en función de los perfiles académicos y profesionales. Se entienden por competencias, aquellos conocimientos relativos a la actividad profesional que son resultado del aprendizaje.

Varias de las competencias contempladas en los planes de estudio de las titulaciones de Grado en el ámbito de las Bellas Artes hacen referencia a las capacidades espaciales y al uso de tecnologías para la interpretación visual.

Por otra parte, el Libro Blanco para el Grado de Bellas Artes (Aneca, 2004) especifica que hay que desarrollar programas de apoyo docente destinados a formar al alumnado dentro de las competencias específicas sobre las que se detecten insuficiencias de formación. Es preciso, por tanto, diseñar pequeños talleres modulares que permitan formar a los alumnos teniendo en cuenta que no todos llegan a la universidad con el mismo nivel de competencias.

En los programas de las asignaturas de estos nuevos Grados no aparecen actividades dirigidas explícitamente a la mejora de las capacidades, como puede ser la elección de sistema de representación o la visión espacial. Se entiende que al cursar asignaturas relacionadas con el espacio, volumen y al conocer los sistemas de representación 
se desarrolla dicha capacidad, aunque no suele medirse su efecto. Por otro lado hay pocos estudios en el ámbito de Bellas Artes que incidan sobre el pensamiento espacial de los alumnos y la mejor estrategia para desarrollarlo (Giménez Morell, Grassa Miranda \& Vidal Alamar, 2010).

En este sentido, proponemos un taller de modelado 3D dirigido a los contenidos de las asignaturas de Sistemas de Representación (vistas normalizadas, perspectivas,..), midiendo el efecto obtenido en los alumnos sobre la capacidad de visión espacial y valorando mediante una encuesta, la eficacia, eficiencia y satisfacción del taller.

\section{Antecedentes}

El Ministerio de Ciencia e Innovación publicó en el Boletín Oficial del Estado (BOE 18,19 y 20 de febrero de 2009) una Orden Ministerial para cada título universitario en el que aparecen las competencias a adquirir los estudiantes para habilitarles en el ejercicio de la profesión.

El libro blanco del grado de BBAA especifica algunas competencias que son genéricas para todas las facultades, como por ejemplo:

1. Capacidad de documentar y determinar el sistema de representación adecuado a la producción artística

2. Capacidad de aplicar profesionalmente tecnologías específicas (libro Blanco)

Adicionalmente, cada centro universitario incluye, al realizar las guías docentes de las asignaturas del grado, otras competencias denominadas específicas para el desarrollo de los objetivos propuestos. Entre ellas podemos destacar:

1. Adquisición de destrezas gráfica y manual y visión espacial. (Facultad de Bellas Artes, Altea-Alicante)

2. Capacidad para la utilización de las técnicas de representación manual e informáticas más adecuadas. (Facultad de BBAA, Altea-Alicante)

3. Capacidad para la visión espacial. (Universidad de Vigo)

4. Habilidad para trabajar en la bidimensionalidad y la tridimensionalidad (Universidad de Vigo)

5. Incrementar la capacidad visual perceptiva. (Universidad Politécnica de Valencia)

El uso, por tanto, de nuevas herramientas tecnológicas y la mejora de la visión espacial son dos capacidades importantes para el alumnado de los nuevos grados de Bellas Artes.

Numerosos estudios demuestran que las habilidades espaciales se pueden desarrollar a través de la formación si se proporcionan los materiales apropiados (Cohen, Hegarty, Keehner, \& Montello, 2003), (Kinsey, 2003), (Newcomer, Raudebaugh, Mckel, \& Kelley, 1999), (Potter \& Van der Merwe, 2003), y existe unanimidad acerca de que la capacidad espacial se puede mejorar mediante el entrenamiento (Sorby, Wyssocky, \& Baartmans, 2003). 
Como herramientas tecnológicas asociadas a los sistemas de representación, los programas de diseño asistido por ordenador han sido empleados tradicionalmente en ámbitos educacionales afines a la ingeniería, sin extenderse su uso en materias relacionadas con los estudios de Bellas Artes.

En la Universidad de La Laguna, coincidiendo con la implantación del Grado en Bellas Artes, decidimos introducir en la docencia reglada de la asignatura Sistemas de Representación, un taller de modelado 3D dirigido a la interpretación y análisis de las formas.

Este taller tiene como objetivo medir su influencia sobre la mejora de las capacidades de los alumnos. Si bien en el dibujo tipo croquis hay debate sobre si las herramientas digitales deben sustituir a los métodos tradicionales de dibujo (Ruiz Llamas, 2003), cuando se habla de sistemas de representación, el uso de herramientas de diseño asistido por ordenador permite al alumno no sólo visualizar sino también hacer diferentes pruebas con sistemas o vistas, hasta encontrar aquella que mejor se ajusta a su dibujo.

Dicho taller ya había sido probado en estudios de Ingeniería (Martin-Dorta, Saorin \& Contero, 2008) y para utilizarlo en la facultad de Bellas se rediseñó por completo al objeto de hacerlo más fácil de implantar (De la Torre, Saorín, Carbonell, Martín-Dorta \& Contero, 2011). El aula donde se impartían las clases no disponía de ordenadores ni de red wifi que permitiera conectar a un aula virtual, por lo que la implementación del taller requería el uso de los ordenadores de los alumnos y una planificación adecuada.

En este artículo vamos a describir la experiencia de un taller de modelado 3D para el análisis y representación de las formas y su efecto en la mejora de las capacidades, destacando por su importancia las habilidades espaciales.

\section{Las habilidades espaciales y su medición}

La capacidad espacial es aceptada por distintos autores a través de la historia como un componente de la inteligencia. Algunas personas disponen de un mayor grado de aptitud innata, pero la gran mayoría puede entrenar esta habilidad a través de la práctica (Sorby et al., 2003). En este trabajo empleamos el término de habilidad espacial para referirnos a la parte de la capacidad espacial que podemos adiestrar mediante entrenamiento.

A la hora de estudiar los componentes de la habilidad espacial encontramos distintos enfoques para establecer su clasificación y, a su vez, varias herramientas para obtener resultados cuantitativos a través de la utilización de test específicos. (Linn \& Petersen, 1985). Es frecuente encontrar las habilidades espaciales divididas en dos subhabilidades como son las Relaciones Espaciales o Rotación Mental, (habilidad de rotar en nuestra imaginación, rápida y acertadamente figuras de dos o tres dimensiones) y la Visión Espacial, (habilidad de reconocer piezas tridimensionales mediante plegado y desplegado de sus caras).

Son muchos los test cuyo objetivo es medir las habilidades espaciales. Cada uno de los test mide alguna de las componentes en los que se clasifican las habilidades espaciales. En nuestra investigación utilizaremos uno de ellos, el Mental Rotation 
Test (MRT) (Albaret and Aubert, 1996; Vanderberg \& Kuse, 1978) que mide la rotación mental y que ha sido validado por trabajos de campo y utilizados en estudios similares al nuestro (Devon, Engle, Foster, Sathianathan, \& Turner, 1994; Sorby \& Baartmans, 2000; Gerson, Sorby, Wisocki \& Baartmans, 2001; Martín-Dorta et al., 2008). La máxima puntuación a obtener en este test es de 40 puntos. (Fig.1).
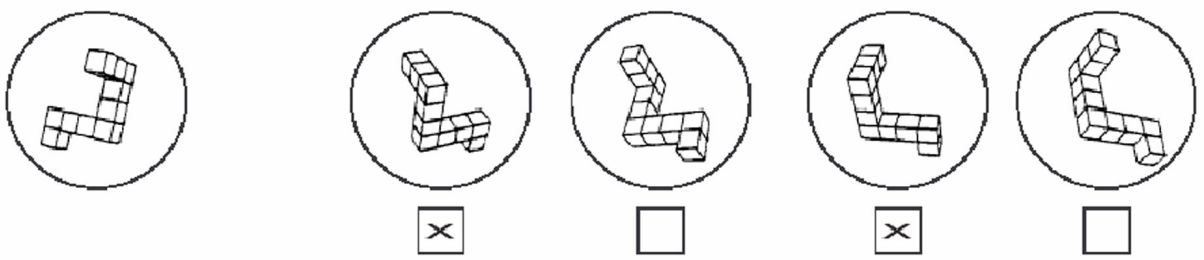

Figura 1. Ejemplo de ejercicio del Mental Rotation Test (MRT). (Albaret and Aubert, 1996; Vanderberg \& Kuse, 1978)

\section{Prueba piloto: Taller de modelado 3D}

\subsection{Participantes}

En nuestro taller de modelado 3D han participado estudiantes del segundo curso del Grado Bellas Artes de la Universidad de La Laguna en la asignatura de Técnicas de Representación durante el curso académico 2010-2011. Han participado un total de 40 estudiantes ( 14 hombres y 26 mujeres) con una media de edad de 25 años. Para el $65 \%$ de los participantes era la primera vez que recibían un curso de modelado tridimensional.

\subsection{Hardware y software}

El aula donde se realizó el taller no disponía de ordenadores ni de red wifi, por lo que se solicitó a los alumnos que trajeran su propio ordenador portátil. Los archivos con el software y los ejercicios se distribuyeron en un pendrive para su instalación individual.

El software elegido para nuestro estudio es el Google SketchUp versión 8, una aplicación multimedia de libre acceso, multiplataforma (PC y Mac) y de descarga gratuita que nos ofrece la posibilidad de introducirnos en el Modelado 3D con pocos conocimientos y en muy poco tiempo. Dispone de una interface amigable, con un reducido número de órdenes muy intuitivas unido a una sencillez de manejo que propicia un rápido aprendizaje. Su gratuidad facilita la implantación en cualquier centro, eliminando el problema del coste de adquisición de licencias de software.

Con Google SketchUp podemos visualizar los modelos tridimensionales en perspectiva axonométrica o cónica. También permite, dado un modelo, obtener automáticamente sus vistas normalizadas (planta, alzado, perfil) por lo que es una herramienta adecuada para que los alumnos puedan desarrollar la capacidad de documentar y determinar el sistema de representación adecuado a la producción artística. 


\subsection{Medida}

En este taller se pretende medir el impacto del taller en varias de las competencias que afectan a los estudiantes de Bellas Artes. En concreto se pretende obtener datos sobre las habilidades de visión espacial, la capacidad de seleccionar el sistema de representación y uso de tecnologías de la información.

Para medir las habilidades espaciales: cada participante realiza el test MRT antes y después de llevar a cabo el experimento, al objeto de poder evaluar los resultados.

El resto de medidas no se van a tomar de manera directa sino que obtendremos datos de la encuesta de satisfacción que cada participante rellena al finalizar el taller. La encuesta consta de 50 preguntas organizadas de acuerdo a cinco variables: la primera referida a los materiales del taller, la segunda respecto a los contenidos, la tercera acerca de la aplicación Google SketchUp y la cuarta y la quinta sobre la opinión del taller y de los materiales educativos digitales.

Las preguntas realizadas en la encuesta han sido las siguientes:

\section{MATERIAL TALLER MODELADO 3D}

\section{$\mathbf{N}^{\mathbf{0}}$ Pregunta}

1. El material del taller tiene buena y cuidada presentación (figuras de aluminio, ficheros pdf de explicación vistas y guía de referencia,..)

2. La estructura del taller en dos fases (Iniciación y Perfeccionamiento) y cada fase en tres niveles, es adecuada.

3. Es sencillo ubicarte en el taller debido al diseño modular por fases de aprendizaje y niveles de complejidad.

4. La utilización de modelos físicos de aluminio para introducirnos en el Modelado 3D es adecuada.

5. El uso de modelos físicos con las caras coloreadas ha servido para facilitar la realización de los modelos 3D con Google SketchUp.

6. El grado de dificultad de los modelos físicos de aluminio ha sido adecuado.

7. La elección de la Aplicación Google SketchUp para los objetivos del taller ha sido acertada.

8. La presentación del enunciado en el fichero de trabajo ha permitido trabajar de forma más cómoda en el ejercicio planteado.

9. Los materiales del taller son suficientes para asimilar los contenidos.

10. Me parece adecuado que el material del taller sirva para PC y Mac así como para formato de pantalla panorámica y $4: 3$

\section{CONTENIDOS TALLER MODELADO 3D}
$\mathbf{N}^{0}$ Pregunta
1. El número de ejercicios propuestos es suficiente para las horas de trabajo propuestas.
2. Me he sentido capaz de resolver los ejercicios planteados.
3. Me ha dado tiempo de realizar los ejercicios planteados.
4. El contenido del taller me ha resultado claro.
5. He entendido bien los ejercicios de este taller.
6. La diferenciación de los distintos niveles haciendo uso del color me ha resultado adecuada.
7. El grado de dificultad de los ejercicios lo considero adecuado en cada nivel. 
8. Realizando los ejercicios, los he resuelto mejor de lo que esperaba.

9. Los contenidos de este taller me han permitido entender mejor las vistas normalizadas.

10. Los contenidos de este taller me han servido para aprender a modelar objetos tridimensionales diferentes a las figuras propuestas.

\section{APLICACIÓN GOOGLE SKETCHUP}

\section{$\mathbf{N}^{0}$ Pregunta}

1. La aplicación Google SketchUp ha mejorado mi visión espacial.

2. Google SketchUp me ha resultado fácil de aprender a utilizar.

3. La presentación de los ejercicios en el formato de Google SketchUp ha sido amigable.

4. He aprendido rápido a utilizar la aplicación Google SketchUp.

5. Las herramientas disponibles en Google SketchUp han sido suficientes para la realización de ejercicios propuestos.

6. La barra de herramientas vistas (isométrica, planta, frontal, derecha, posterior, izquierda) ha sido importante para la mejor comprensión espacial de los ejercicios.

7. Podría aprender a utilizar Google SketchUp sin necesidad de explicaciones por parte de la profesora/profesor

8. Es una gran ventaja que exista versión gratuita de Google SketchUp para PC y para Mac

9. ¿Consideras que Google SketchUp te puede servir en el desempeño de tu carrera?

10. ¿Recomendarías Google SketchUp a tus compañeros?

\section{OPINIÓN TALLER MODELADO 3D}

\section{$\mathbf{N}^{\mathbf{0}}$ Pregunta}

1. ¿Crees que el taller realizado cumple con la finalidad para la que ha sido planteado (Análisis y representación de las formas a través de modelado 3D)?

2. El taller realizado ¿te parece útil para mejorar el nivel de visión espacial?

3. ¿Crees que es necesario material didáctico adicional para realizar el taller?

4. Para realizar este taller, ¿podrías haber trabajado de forma autónoma? Es decir sin necesidad de asistencia del profesor.

5. ¿Crees que este tipo de talleres puede mejorar tu atención y motivación para el estudio de los contenidos de asignaturas relacionadas con el análisis y representación de las formas?

6. La diferenciación de los distintos niveles haciendo uso del color me ha resultado adecuada.

7. ¿Crees que el taller se puede desarrollar en cualquier momento y fuera del aula de clase (en casa, en el autobús, en el tranvía, etc.)?

8. Creo que el uso de ejercicios desarrollados con SketchUp es una buena herramienta para el desarrollo de la capacidad espacial y el aprendizaje de las vistas normalizadas.

9. ¿Crees que es necesario material teórico adicional para realizar los ejercicios de este taller?

10. ¿Crees que el uso de herramientas $3 \mathrm{D}$ puede mejorar tu atención y motivación para el estudio de los contenidos de las asignaturas de Expresión Gráfica? 


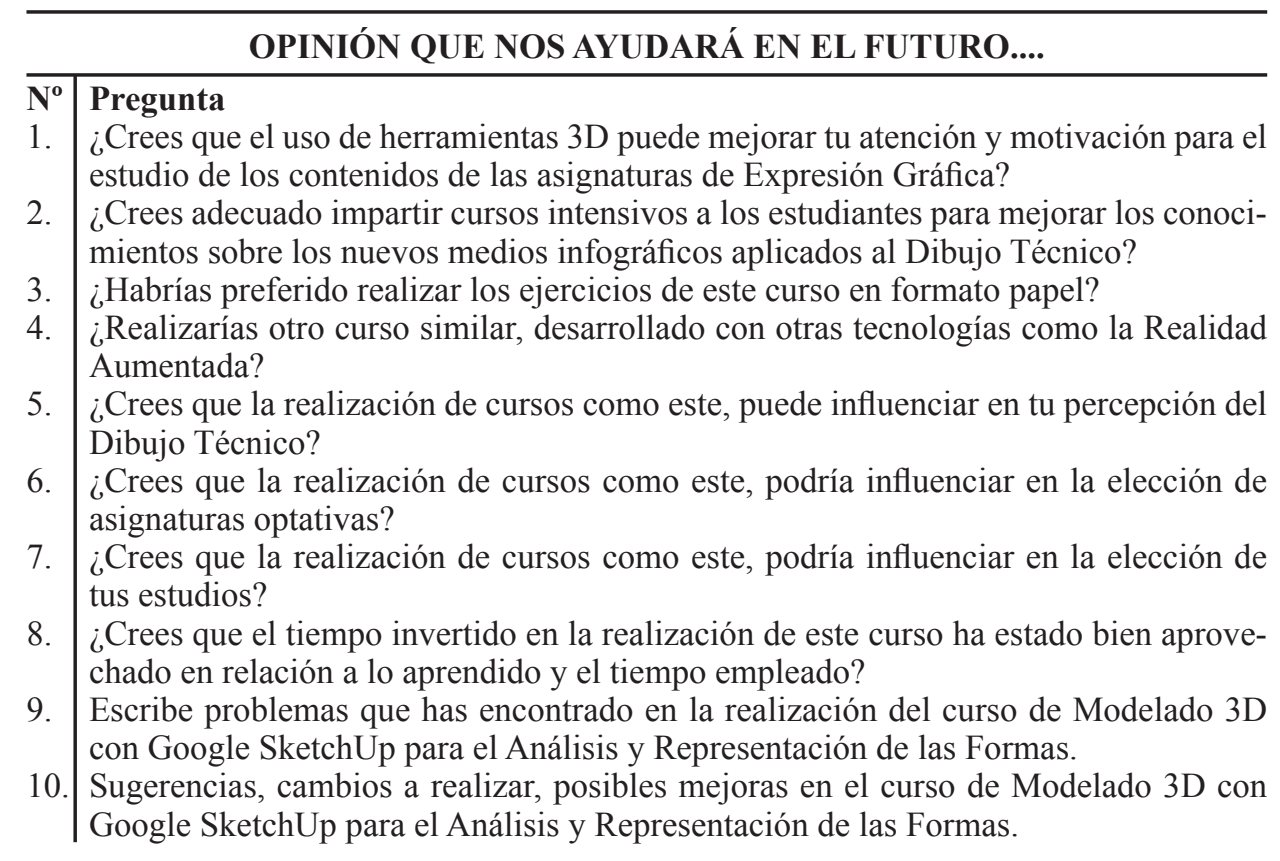

Las preguntas se han realizado utilizando una escala tipo Likert, según la cual, a cada pregunta el encuestado le asigna una valoración numérica indicando el grado de acuerdo o desacuerdo con respecto a la pregunta planteada en una escala de 5 puntos.

\subsection{Instrucción}

Este taller propone una estrategia que combina la manipulación de piezas reales e imágenes tridimensionales de ordenador. (Martin-Dorta et al., 2008)

De manera separada, esta estrategia ha sido validada como instrumento de mejora de habilidades espaciales por otros investigadores: algunos han trabajado con piezas reales (Alias, Black \& Gray, 2002; Ben-Chaim, Lappan \& Hougang, 1998; Duesbury \& O'Neil, 1996) y otros concluyen que manipular la imagen de un objeto por ordenador es suficiente para mejorar las habilidades espaciales (Wiley 1990; Sorby, 1999). El taller consta de dos fases: Iniciación y Perfeccionamiento. Existen a su vez tres niveles de dificultad que se consiguen con la incorporación de caras inclinadas y caras curvas. A su vez, las figuras están dibujadas en una rejilla tridimensional que aumenta su complejidad con el nivel. Cada nivel contiene 24 figuras. (Figura 2)

El diseño de este taller ha sido validado por el Grupo de Investigación DEHAES (http://www.degarin.com/dehaes/) de la Universidad de La Laguna durante el curso 2010-2011, comprobando cuantitativamente la adecuada distribución en fases de aprendizaje y niveles de dificultad: se han obtenido unos tiempos medios de resolución de ejercicios por fases y niveles de 3 minutos para la fase de iniciación y de 8 minutos para la fase de perfeccionamiento. 
En la fase de Iniciación, el alumno, siguiendo las instrucciones del video tutorial incluido en el taller realiza un entrenamiento básico del manejo del software SketchUp. Los estudiantes aprenden las funciones más importantes del programa, como por ejemplo dibujar líneas y polígonos. También aprenden operaciones básicas de modelado, como por ejemplo hacer extrusiones.

Una vez familiarizados con el programa realizan la práctica 1.1, consistente en crear modelos 3D a partir de piezas reales de aluminio. Para esta fase se han empleado cinco juegos del maletín M14 (lote14A) de la empresa Maditeg (Maditeg, 1997) consistente en 30 piezas mecanizadas de aluminio. Posteriormente, el alumno accede a la práctica 1.2 , en la que ha de crear modelos $3 \mathrm{D}$ a partir de perspectivas isométricas de figuras, con tres grados de dificultad creciente.

\begin{tabular}{|c|c|c|c|c|}
\hline FASE & \multicolumn{2}{|c|}{ PRÁCTICAS/NIVEL } & LOGO & DESCRIPCIÓN \\
\hline \multirow{4}{*}{ 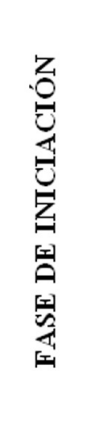 } & \multicolumn{2}{|c|}{ PRÁCTICA 1.1} & & $\begin{array}{c}\text { Crear modelos } 3 \mathrm{D} \text { a partir de piezas } \\
\text { reales de aluminio. }\end{array}$ \\
\hline & \multirow{3}{*}{\begin{tabular}{|c} 
PRÁCTICA \\
$\mathbf{1 . 2}$ \\
Crear \\
modelos 3D a \\
partir de \\
perspectivas \\
isométricas de \\
figuras
\end{tabular}} & NIVEL A & & $\begin{array}{c}24 \text { figuras inscritas en una rejilla de } \\
3 \times 3 \times 3 \text { con sus caras paralelas a los } \\
\text { planos coordenados }\end{array}$ \\
\hline & & NIVEL B & & $\begin{array}{l}24 \text { figuras inscritas en una rejilla de } \\
4 \times 4 \times 4 \text { que además incluyen caras } \\
\text { inclinadas }\end{array}$ \\
\hline & & NIVEL C & & $\begin{array}{c}24 \text { figuras inscritas en una rejilla de } \\
5 \times 5 \times 5 \text { que además incluyen caras curvas }\end{array}$ \\
\hline \multirow{3}{*}{ 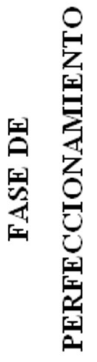 } & \multirow{3}{*}{\begin{tabular}{|c} 
PRÁCTICA \\
$\mathbf{2 . 1}$ \\
Crear \\
modelos 3D a \\
partir de las \\
vistas \\
normalizadas \\
de figuras
\end{tabular}} & NIVEL A & & $\begin{array}{c}24 \text { figuras inscritas en una rejilla de } \\
3 \times 3 \times 3 \text { con sus caras paralelas a los } \\
\text { planos coordenados }\end{array}$ \\
\hline & & NIVEL B & & $\begin{array}{l}24 \text { figuras inscritas en una rejilla de } \\
4 \times 4 \times 4 \text { que además incluyen caras } \\
\text { inclinadas }\end{array}$ \\
\hline & & NIVEL C & & $\begin{array}{c}24 \text { figuras inscritas en una rejilla de } \\
5 \times 5 \times 5 \text { que además incluyen caras curvas }\end{array}$ \\
\hline
\end{tabular}

Figura 2. Diseño del Taller de Modelado 3D

En la fase de perfeccionamiento el alumno realiza la práctica 2.1, consistente en crear modelos 3D a partir de vistas ortogonales normalizadas de figuras. En esta fase los alumnos reciben indicaciones teóricas sobre los principios generales de representación, en concreto sobre vistas normalizadas al objeto de poder abordar este nivel. Como en la práctica 1.2, esta fase está también estructurada en tres niveles A, B y C en grado creciente de dificultad.

Para la realización de los ejercicios se busco diseñar entornos de trabajo controlados donde junto con el enunciado el alumno dispusiera de una rejilla de referencia que les ayudara en la realización de los modelos tridimensionales. (Figura 3) 


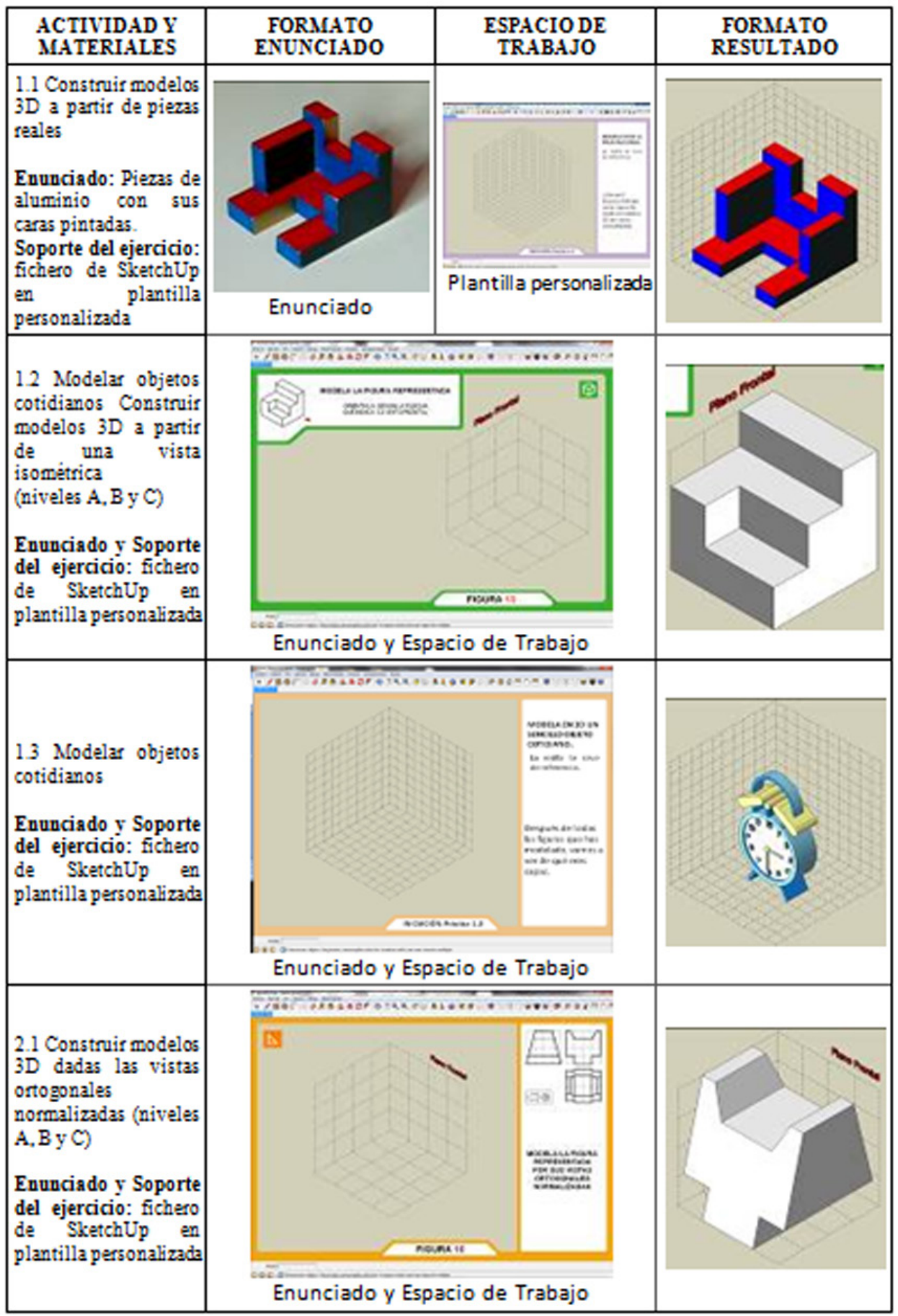

Figura 3. Diseño del Taller de Modelado 3D 
El taller se impartió en 3 sesiones de 2 horas cada una, de acuerdo a la siguiente tabla:

\begin{tabular}{|l|l|l|l|l|}
\hline & & $\begin{array}{l}\text { Duración } \\
\text { (minutos) }\end{array}$ & Sesión \\
\hline \multirow{4}{*}{$\begin{array}{l}\text { Fase } \\
\text { Iniciación }\end{array}$} & $\begin{array}{l}\text { Instalación Software y } \\
\text { Ejercicios del curso }\end{array}$ & 30 & \multirow{2}{*}{ Sesión 1 } \\
\cline { 2 - 3 } & Entrenamiento Básico & 30 & \\
\cline { 2 - 3 } & Piezas Reales & 60 & \\
\cline { 2 - 3 } & Nivel A & 30 & \multirow{3}{*}{ Sesión 2 } \\
\cline { 2 - 3 } & Nivel B & 30 & \\
\cline { 2 - 3 } & Nivel C & 60 & \\
\hline Fase \\
Perfeccionamiento & Nivel A & 30 & \\
\cline { 2 - 3 } & Nivel B & 45 & \\
\cline { 2 - 3 } & Nivel C & 45 & \\
\hline
\end{tabular}

Tabla 1. Temporalización del taller.

Gracias a la modularidad del taller se puede implementar en cualquier programa docente adaptando el número de ejercicios a desarrollar en función del nivel del alumno y del número de horas de que disponga la asignatura.

\subsection{Hipótesis de trabajo}

Las hipótesis de trabajo de las que partimos son las siguientes:

1. Un taller de modelado 3D es una herramienta válida para el objetivo de mejorar las habilidades espaciales.

2. El taller es útil para el aprendizaje de la asignatura de sistemas de representación.

3. El empleo de nuevas herramientas tecnológicas de aprendizaje mejora la motivación del alumno.

Para poder validar la hipótesis 1 se fijará una hipótesis nula $(\mathrm{H} 0)$ y se validará o no la suposición a través de métodos de inferencia estadística. Para la hipótesis 2 y 3 , se aportan datos de encuestas de satisfacción cumplimentadas por los participantes al finalizar el taller. 


\section{Análisis de los datos}

\subsection{Habilidades espaciales}

De la encuesta de satisfacción se obtiene que el 95\% de los participantes indican que el uso de ejercicios desarrollados con SketchUp es una buena herramienta para el desarrollo de la capacidad espacial. Esta apreciación de los alumnos la podemos verificar gracias a los resultados obtenidas por en los test de visión espacial.

La tabla 2 muestra las puntuaciones medias obtenidas por los estudiantes antes (Pre) y después (Post) de realizar el taller, así como las ganancias medias, para el test MRT.

\begin{tabular}{|l|c|c|c|c|}
\hline & \multicolumn{4}{|c|}{ MRT } \\
\hline $\begin{array}{l}\text { Curso } \\
\text { 2010-11 }\end{array}$ & $\begin{array}{c}\text { Total } \\
\mathrm{N}=40\end{array}$ & $\begin{array}{c}\text { Pre } \\
\text { (s.d.) }\end{array}$ & $\begin{array}{c}\text { Post } \\
\text { (s.d.) }\end{array}$ & $\begin{array}{c}\text { Gain } \\
\text { (s.d.) }\end{array}$ \\
\hline & & 15,70 & 22.68 & 6.98 \\
& & $(7.54)$ & $(9.93)$ & $(5.09)$ \\
\hline
\end{tabular}

Tabla 2. Resultados del pre y post test MRT.

Para el análisis estadístico usamos la variable t-student (Student's t-test), partiendo de la hipótesis nula (H0): los valores medios de las habilidades espaciales no han variado después del entrenamiento. Se aplica la prueba t-Student para series emparejadas y se obtienen los p-valor de $\mathrm{P}=6,29413 \mathrm{E}-11$.

Se comprueba que el nivel de significación no llega en ningún caso al $1^{\mathrm{o} / \mathrm{oo}}$, por lo que se rechaza la hipótesis nula en todos los casos y podemos afirmar, con un nivel de significación superior al 99,9\%, que la variación media del grupo estudiado ha experimentado un aumento. Es decir, que existe un efecto del taller sobre el valor medio de las habilidades espaciales medidas en los participantes sometidos al entrenamiento a través del taller de modelado 3D.

\subsection{Aprendizaje de Sistemas de Representación}

De la encuesta de satisfacción se obtiene que:

- Un $85 \%$ de los alumnos cree que ha sido provechoso este curso para la asignatura Sistemas de Representación.

- El 95\% indica que el uso de ejercicios desarrollados con SketchUp es una buena herramienta para el aprendizaje de las vistas normalizadas.

- Los contenidos de este curso me han permitido entender mejor las vistas normalizadas (3,95 sobre 5 en escala de likert) 


\subsection{Empleo de nuevas herramientas y motivación}

La medición de la motivación se puede hacer mediante encuesta de opinión o mediante consultas indirectas, de tal manera que se evalúe si los participantes realizan más ejercicios de los propuestos, etc.. En nuestra prueba hemos valorado el uso de herramientas digitales enlazado con la motivación de los alumnos mediante la encuesta de satisfacción y preguntas directas para evaluar su opinión sobre los materiales del taller de modelado. Se han obtenido los siguientes resultados:

- El 83\% de los participantes cree que este taller mejora su atención y motivación para el estudio de contenidos referentes al análisis, diseño e interpretación de las formas.

- Los participantes estiman que han realizado el taller mejor de lo que esperaban con un valor de 3,67 sobre 5 .

- El 78\% opina que el tiempo invertido en la realización de este curso ha estado bien aprovechado en relación a lo aprendido.

- El $77 \%$ prefiere el formato basado en materiales digitales frente al formato tradicional de papel.

\section{Conclusiones y futuros trabajos}

De la experiencia obtenida en la realización del taller de modelado 3D podemos concluir, respecto de las hipótesis planteadas:

- Hipótesis 1: Las habilidades se pueden desarrollar mediante entrenamiento. El taller de modelado 3D utilizando Google SketchUp se ha mostrado como una buena opción para este propósito: ha aumentado significativamente la capacidad espacial de los participantes con una ganancia media de 6,98 puntos en el test MRT.

- Hipótesis 2: El taller, de acuerdo a los resultados de la encuesta de satisfacción, sirve para el aprendizaje de los sistemas de representación y vistas normalizadas.

- Hipótesis 3: El empleo de nuevas herramientas tecnológicas de aprendizaje mejora la motivación del alumno (un $83 \%$ de los alumnos así lo consideran) y mayoritariamente (77\%) prefieren el formato digital al papel.

En la línea de futuros trabajos:

- Sería interesante adaptar los contenidos de este taller a los nuevos soportes móviles como las tabletas digitales de reciente aparición. 
- El uso de piezas reales de aluminio en la primera práctica del taller es muy formativo pero no se puede digitalizar. Por ello proponemos sustituir esa práctica por un conjunto de piezas iguales disponibles mediante el uso de realidad aumentada.

- Sería interesante la implementación de estos talleres dentro de un aula virtual.

- De acuerdo a los resultados de la encuesta, nos proponemos desarrollar nuevos materiales educativos digitales que combinen dos y tres dimensiones para realizar prácticas específicas de la asignatura.

\section{Referencias}

Albaret, J.M. \& Aubert, E. (1996). Etalonnage 15-19 ans du test de rotation mentale de Vanderberg. Evolutions Psychomotrices, 8 (34), 268-278.

Alias, M.; Black, T., \& Gray, D. (2002). Attitudes Towards Sketching and Drawing and the Relationship with Spatial Visualization Ability in Engineering Students. International Education Journal, 3 (3), 165-175.

Aneca. (2004). Libro Blanco para el diseño de la titulación de Grado: Bellas Artes. Barcelona: Agencia Nacional de Evaluación de la Calidad y Acreditación.

Ben-Chaim, D.; Lappan, G. \& Hougang R.T. (1998). The effect of instruction on spatial visualization skills of middle school boys and girls. American Educational Research Journal , 25 (1), 51-71.

Cohen, C.; Hegarty, M. \& Montello, D. (31 -2 de July-August de 2003). Spatial Ability in the Representation of Cross Sections. Proceeding of the 25th Annual Conference of Cognitive Science Society, 1333-1334.

De la Torre Cantero, J.; Saorín, J.L.; Carbonell, C.; Martín-Dorta, N. \& Contero, M. (2011).Rediseño, digitalización y validación de un curso intensivo para la docencia en el análisis y estudio de las formas. VIII Multidisciplinary Symposium on Design and Evaluation of Digital Content for Education, (SPDECE), 154158.

Devon, R.; Engle, R.; Sathianathan, D. \& Turner, G. (1994). The Effect of Solid Modeling Software on 3D Visualization Skills. Engineering Design Graphics Journal, 58 (2), 4-11.

Duesbury, R.T. \& O'Neil, H.F. (1996). Effect of practice in a computer-aided design environment in visualizing three-dimensional objects from two-dimensional orthographic projections. Journal of Applied Psychology, 81 (3), 249-260.

Gerson, H.; Sorby, S.; Wysocki, A. \& Baartmans, B. (2001). The Development and Assessment of Multimedia Software for Improving 3-D Spatial Visualization Skills. Computer Applications in Engineering Education, 9 (2), 105-113.

Giménez Morell, R.; Grassa Miranda, V. \& Vidal Alamar, M. (2010). Consideraciones sobre las imágenes mentales en el sistema diédrico español. Arte, Individuo y Sociedad (1), 111-120.

Kinsey, B. (2003). Design of a CAD Integrated Physical Model Rotator. Annual Conference \& Exposition Engineering Education, on line, Avaiable from www. ni.com/academic/journal_asee.htm. 
Linn, M. \& Petersen, A. (1985). Emergence and Characterization of Sex Differences in Spatial Ability: A Meta-Analysis. Child Development, 56 (6), 1479-1498.

Maditeg. (1997). Didactic Material for Engineering Design Graphics: M14 briefcase: Modelos de tres vistas y cortes.

Martín-Dorta, N.; Saorín, J.L. \& Contero, M. (2008). Development of a Fast Remedial Course to Improve the Spatial Abilities of Engineering Students. Journal of Engineering Education, 97 (4), 505-513.

Newcomer, J.; Raudebaugh, R.; McKell, E. \& Kelley, D. (1999). Visualization, Freehand Drawing, Solid Modeling, and Design in Introductory Engineering Graphics. The 29th ASEE/IEEE Frontiers in Education Conference, on line. Avaiable from http://fie.engrng.pitt.edu/fie99/papers/1006.pdf.

Potter, C. \& Van der Merwe, E. (2003). Perception, imagery, visualization and engineering graphics. Europen Journal of Engineering Education, 28 (1), 117133.

Ruiz Lamas, G. (2003). Problemas del Diseño en la Era de la Digitalización. Arte, Individuo y Sociedad (15), 11-21.

Sorby, S. \& Baartmans, B.J. (2000). The development and assessment of a course for enhancing the 3-D spatial visualization skills of first year engineering students. Journal of Engineering Education, 89 (3), 301-307.

Sorby, S. (1999). Spatial Abilities and Their Relationship to Computer Aided Design Instruction. Proceeding of the 1999 American Society for Engineering Education Annual Conference \& Exposition.

Sorby, S.; Wysocki, A. \& Baartmans, B. (2003). Introduction to 3D Spatial Visualization: an active approach. Clifton Park, NY: Thomson: Delmar Learning.

Vandenberg, S. \& Kuse, A. (1978). Mental Rotations: A Group Test of ThreeDimensional Spatial Visualisation. Perceptual and Motor Skills , 47 (6), 599-604.

Wiley, S.E. (1990). Computer Graphics and the development of visual perception in engineering graphics curricula. Engineering Design Graphics Journal, 54 (3), 30-35. 